\title{
DEVELOPMENT AND EVALUATION OF AN IN SITU THERMOGELLING SYSTEM OF OFLOXACIN FOR CONTROLLED OCULAR DELIVERY
}

\author{
ANANTH PRABHU, MARINA KOLAND* \\ Department of Pharmaceutics, N.G.S.M Institute of Pharmaceutical Sciences, Nitte (Deemed to be University), Mangalore, Karnataka, India. \\ Email: marinakol@nitte.edu.in
}

Received: 8 December 2018, Revised and Accepted: 06 February 2019

\section{ABSTRACT}

Objective: The purpose of this study was to develop an in situ ocular gel of ofloxacin which aimed to prolong corneal residence time while controlling drug release.

Method: In situ gelling solutions were prepared from Poloxamer 407, a temperature-sensitive gelling polymer and to which, mucoadhesive polymers such as hydroxypropyl methyl cellulose 15 cps and polyvinyl alcohol (PVA) were included to provide corneal adhesion. Drug incorporated gels were prepared and evaluated for their appearance, $\mathrm{pH}$, gelation temperature, and in vitro drug release studies.

Results: Incorporation of the drug into the formulation increased the gelation temperature while the addition of mucoadhesive polymers decreased the gelation temperature. Increasing the concentration of bio-adhesive polymers retarded the release of ofloxacin from the poloxamer solutions and drug release was sustained over a period of $9 \mathrm{~h}$. PVA had no significant effect on the gelation temperature and could not sustain the drug release for a longer duration. The in vitro release profiles of the drug from all the formulations could be best expressed by Higuchi's equation which indicated that gels followed matrix diffusion process and drug release from gel formulations followed first-order process.

Conclusion: The results showed that the developed system would be promising in the treatment of ocular infections with the combined advantages of ease of administration, the accuracy of dosing, increased bioavailability, and prolonged retention time.

Keywords: Ofloxacin, Poloxamer 407, Gelation temperature, Muco-adhesive polymers.

(C) 2019 The Authors. Published by Innovare Academic Sciences Pvt Ltd. This is an open access article under the CC BY license (http://creativecommons. org/licenses/by/4. 0/) DOI: http://dx.doi.org/10.22159/ajpcr.2019.v12i3.31233

\section{INTRODUCTION}

When eye drops are administered, the drug dose instilled undergoes drainage immediately upon instillation, i.e., within 5 min which is typical of ophthalmic solutions. Therefore to achieve the desired therapeutic effect, frequent instillation of solutions is required. Due to short precorneal residence time, ophthalmic bioavailability of only $1 \%-10 \%$ is achieved. Systemic absorption of the drug drained through the nasolacrimal duct may result in some undesirable side effects. To overcome these problems and to extend the ocular residence time of medications for topical application to the eye, ophthalmic in situ gels have been investigated. In situ gel formation occurs due to one or combination of different stimuli such as $\mathrm{pH}$ change, temperature modulation, and solvent exchange. Smart polymeric systems represent promising means of delivering the drugs. Once administered these polymers undergo sol-gel transition. These biodegradable polymers have a wide range of clinical applications. Various natural and synthetic polymers are used for the development of in situ drug delivery systems [1].

The poloxamers or pluronics are biodegradable, nonionic triblock copolymers of ethylene oxide and propylene oxide capable of forming thermo-reversible gels. Pluronic F 68 and Pluronic F 127 have been approved by the Food and Drug Administration for use in the formulation of oral solutions, suspensions, topicals, inhalations, and nasal dosage forms [2]. They form sols at room temperature and undergo sol-to-gel transition at body temperature. In our investigations, we have used Pluronic F 127, also known as Poloxamer 407, the solutions of which gel at temperatures above $25^{\circ} \mathrm{C}$ [3]. Moreover, the aqueous solutions of this polymer can be easily sterilized by filtration.

The most common route of ocular drug delivery is topical delivery into the cul-de-sac of the eye [4]. The unique anatomy and physiology of the eye offer many challenges to develop effective ophthalmic drug delivery systems.

Ofloxacin is a synthetic fluoroquinolone agent widely used in the treatment of ocular conjunctivitis and other ocular disorders. Conventional eye drops of ofloxacin is a $0.3 \%$ solution of the drug used in a dose of 1-2 drops every 2-4 h in the control of conjunctivitis and for symptomatic relief of pain and inflammation [5].

In the present study, an attempt was made to develop an in situ ocular gelling system of ofloxacin which combines the advantages of conventional eye drops in terms of convenience of administration and dose accuracy and those of ophthalmic inserts in terms of prolonged residence time, extended and more efficient drug effects and decreased frequency of administration leading to better patient compliance.

\section{MATERIALS AND METHODS}

Materials

Ofloxacin was received as a gift sample from Sanofi Aventis Goa. Pluronic F 127 or Poloxamer 407, hydroxypropyl methylcellulose (HPMC) 15 cps and polyvinyl alcohol (PVA) were procured from Yarrow Chem Products, Mumbai. All the other chemicals used were of analytical grade.

\section{Methods}

Various compositions of drug-free in situ gelling solutions were prepared using Poloxamer, HPMC, and PVA in various ratios. The resulting polymer compositions were evaluated for physical characteristics such as appearance, $\mathrm{pH}$, gelation temperature, and gelling capacity. The results of these studies were used to select the optimal compositions which were then incorporated with the drug [6]. 


\section{Preparation of drug incorporated gelling solutions}

The gel-forming solutions were prepared by the cold method as used for the drug-free solutions. Poloxamer was added to half the quantity of distilled water with continuous agitation, and this polymer base was left at $4^{\circ} \mathrm{C}$ for $24 \mathrm{~h}$ to dissolve until a clear solution was obtained. Required percentage of benzalkonium chloride was added as preservative to this polymer base. Mucoadhesive polymers such as PVA and HPMC were dissolved in distilled water which was then added to the poloxamer solution and diluted up to $25 \mathrm{ml}$. The drug was separately dissolved in buffer and was added to the polymer solution and adjusted to the required volume to form a clear solution [7].

\section{Evaluation of drug incorporated in situ gelling solutions}

Appearance and $\mathrm{pH}$

The developed formulations were visually inspected for clarity in sol and gel form.

The $\mathrm{pH}$ of the formulations was determined using the pen $\mathrm{pH}$ meter, by bringing the electrode in contact with the surface of the formulations [8.9].

Viscosity measurement

The viscosity was measured using a Brookfield viscometer and the angular velocity increased gradually from 2 to $50 \mathrm{rpm}$. The studies were performed using spindle no. 96 for gels at physiological temperature $\left(37^{\circ} \mathrm{C}\right)$ and for sols at normal room temperature $\left(28^{\circ} \mathrm{C}\right)$ [10]. The viscosity of measured formulations is given in Tables 1 and 2 .

Measurement of gelation temperature

About $10 \mathrm{ml}$ of the formulation was transferred to a $50 \mathrm{ml}$ beaker with a magnetic bead and placed on a magnetic stirrer with thermostatically controlled heater. The temperature of the stirrer was increased in increments of $1^{\circ} \mathrm{C}$ and the temperature of the formulation was recorded using a thermometer. The rotation of bead gradually slowed down as the viscosity increased. The temperature at which the magnetic bead stopped rotating was taken as gelation temperature [11]

\section{Gelling capacity}

The gelling capacity was determined by placing a drop of the system in a vial containing $2 \mathrm{ml}$ of simulated tear fluid (phosphate buffer of $\mathrm{pH}$ 7.4) freshly prepared and equilibrated at $37^{\circ} \mathrm{C}$ and visually assessing the gel formation and noting the time for gelation and the time taken for the formed gel to dissolve [12]. The following grades were given based on gelling capacity.

- -: No gelation

- +: Gels after a few minutes, dissolves rapidly

- ++: Gelation immediate, remains for a few hours

- +++: Gelation immediate, remains for extended period.

\section{Drug content estimation}

Each formulation $(8.3 \mathrm{ml})$ was taken in a $100 \mathrm{ml}$ volumetric flask diluted with Phosphate buffer of $\mathrm{pH} 7.4$ and shaken to dissolve the drug.
The solution was filtered through Whatman filter paper and $1 \mathrm{ml}$ of the filtered solution was taken and further diluted to $10 \mathrm{ml}$ with artificial tear fluid. Drug content was estimated using an UV spectrophotometer by measuring the absorbance of the above solution at $288 \mathrm{~nm}$. Results are given in Table 3 .

\section{In vitro diffusion studies}

In vitro drug release studies were carried out in Franz-Diffusion (FD) cell using cellophane membrane as diffusion membrane and Phosphate buffer (pH: 7.4) as medium. The cellophane membrane (previously soaked overnight in the receptor medium) was tied at one end of the FD cell. One ml of artificial tear fluid was added to the donor compartment and $1 \mathrm{~mL}$ of the gel was spread uniformly on a cellophane membrane, which was in contact with the receptor medium. The receptor medium was stirred continuously at $20 \mathrm{rpm}$ to simulate sink conditions. The whole assembly was placed on a magnetic stirrer and maintained at 36 $\pm 1^{\circ} \mathrm{C}$ to mimic physiological condition at which formulation remains in gel form. Aliquots of medium were withdrawn at appropriate time intervals, and equal volumes of fresh media were added to replace the withdrawn samples [13]. Withdrawn samples were filtered, diluted, and estimated by ultraviolet (UV) double beam spectrophotometer (UV Spectrophotometer- JASCO) at $288 \mathrm{~nm}$.

\section{Kinetic analysis of drug release data}

The higher values of regression coefficient for the first-order equation indicated that percentage drug release from gel formulations probably followed first-order kinetics. Since it is a gel diffusion process, therefore to understand the mechanism of drug release the drug release data was also fitted to Higuchi's Matrix model [14].

\section{In vitro antimicrobial studies}

When antibiotics are included in the formulations, it is necessary to carry out studies for evaluation against selective microorganisms, the results obtained are compared with that of suitable standards. For the studies, the medium used is the nutrient agar of pH7.4. After sterilization, the medium was kept aside at room temperature. The medium was poured into a sterilized petri dish to give a depth of 3-4 $\mathrm{mm}$ and was ensured that the layer of medium is uniform in thickness. After solidification, a loop of diluted suspension culture (Staphyococcus aureus) in nutrient broth was added on to the surface of solidified agar and was spread uniformly with the help of L-shaped rod. After stabilization of culture, with the help of a sterile cork borer, cups of $6 \mathrm{~mm}$ diameter each were punched and scooped out from the petri dish. Gels of known concentration along with marketed and standard preparation were fed into the cup. The petri dish was then incubated for 24 hat $37^{\circ} \mathrm{C}$. After incubation, the zone of inhibition (ZOI) was measured [15]. The entire operation was carried out in a laminar airflow unit. Each formulation solution was tested in triplicate. Both positive and negative controls were maintained throughout the study.

Table 1: Viscosity of (gel form) after thermo-conversion in cps

\begin{tabular}{llllll}
\hline Formulation code & Viscosity at 2 rpm & Viscosity at $\mathbf{5} \mathbf{~ r p m}$ & Viscosity at 10 rpm & Viscosity at 20 rpm & Viscosity at 50 rpm \\
\hline OG1 & 3543 & 2389 & 1357 & 711.6 & 310.4 \\
OG2 & 2265 & 1368 & 728.5 & 315.6 \\
OG3 & 2645 & 2285 & 1370 & 728 & 319.5 \\
OG4 & 3543 & 1387 & 719.2 & 321 \\
\hline
\end{tabular}

Table 2: Viscosity of formulations (sol form) in cps

\begin{tabular}{llllll}
\hline Formulation code & Viscosity at 2 rpm & Viscosity at $\mathbf{5}$ rpm & Viscosity at 10 rpm & Viscosity at 20 rpm & Viscosity at 50 rpm \\
\hline OG1 & 16.4 & 12.0 & 7.31 & 2.9 & 1.31 \\
OG2 & 16.5 & 14.0 & 7.50 & 2.71 & 1.10 \\
OG3 & 18.7 & 15.0 & 7.5 & 2.81 & 1.12 \\
OG4 & 18.1 & & 2.77 & 1.14 \\
\hline
\end{tabular}




\section{RESULTS AND DISCUSSION}

On the basis of results from the evaluation of the physical characteristics such as appearance, $\mathrm{pH}$, gelation temperature and gelling capacity, the selected compositions were incorporated with the drug. The formulae for the optimal compositions are shown in Table 4. All the formulations developed had a clear appearance in the sol form when stored at room temperature not exceeding $30^{\circ} \mathrm{C}$

The formulations were in the $\mathrm{pH}$ range of 6.6-7.2 which is well within the range specified for ocular formulations and which is considered acceptable to avoid the risk of irritation on application to the eye. This $\mathrm{pH}$ range provides a better environment for mucoadhesion as well as longer retention of formulation in the ocular cavity mentioned in Table 3.

The prepared formulations had an acceptable gelling capacity. Formulations OG2 and OG4 showed an acceptable gelling capacity and retained as a gel for a longer period. The formulations OG1 and OG3 had lesser gelling capacity due to lesser concentration of mucoadhesive polymers along with poloxamer.

Incorporation of PVP in different concentrations had no significant effect on the gelation temperature, but the incorporation of HPMC led to a gradual decrease in the gelation temperature with increasing concentrations of the polymer as shown in Table 3. The results showed that the maximum gelation temperature of the drug formulations did not exceed $32^{\circ} \mathrm{C}$. We can safely assume that the formulations were gelled at body temperature of $37.5^{\circ} \mathrm{C}$ and the inclusion of drug and additives did not appreciably affect the gelation temperature. The percentage drug content of all the prepared formulations was found to be within a range of $96.74 \%$ $94.67 \%$ as shown in Table 3 .

The gels were less viscous in solution form when compared to the gel state. As the rpm increased the viscosity decreased proportionately as shown by results given in Tables 1 and 5 .

In vitro drug diffusion studies demonstrated that all the four formulations showed sustained drug release for $9 \mathrm{~h}$. The combination of the poloxamer with HPMC or PVA showed faster dissolution in $9 \mathrm{~h}$ compared to solutions containing poloxamer alone. The release of the drug was prolonged with increased concentration of poloxamer in the formulation. This is probably because the increased viscosity of the poloxamer only formulation resulted in slower diffusion of the drug from the gel [16]. Formulations containing HPMC were able to show the better release of the drug over a duration of $9 \mathrm{~h}$ due to the lower viscosity of their gels compared to formulations containing the only poloxamer or in combination with PVA which retard the drug release considerably.

All the formulations showed the initial burst effect that may be attributed to the solution form of the formulation at the initial phase of drug release, after which the formulation undergoes thermoconversion to form a gel and resulting in sustained release of the drug from the formulation. Thus the release of the drug could be modulated by adjusting the concentration of the polymers to obtain a sustained drug release profile for $9 \mathrm{~h}$.
The maximum percentage of drug release was found to be $91.40 \%$ in $9 \mathrm{~h}$ from formulation OG2. The in vitro drug release profiles from all the formulations are depicted in Fig. 1.

Kinetic analysis of drug release data show that the diffusion of drug from the formulation took place by first order kinetics since the values for regression coefficient $\left(\mathrm{R}^{2}\right)$ were higher than for the zeroorder model. Since drug release is a gel diffusion process, therefore to understand the mechanism of release, the data was further fitted to Higuchi's Matrix model [16]. The results of the kinetic analysis as shown in Table 5, further supported the presumption that drug release from the gel followed a matrix diffusion process.

Microbial studies were carried out by taking the pure drug as a standard and marketed solution (ofloxacin eye drops, $0.3 \% \mathrm{w} / \mathrm{v}$ ) as reference. Among all the formulations, formulation OG1 showed a maximum ZOI of $24 \mathrm{~mm}$ which was the result of the slow and sustained diffusion of the drug from the formulation due to its greater viscosity. On the other hand, formulations OG2 and OG3 showed significantly smaller ZOI when compared to OG1, OG4, the standard $(0.3 \% \mathrm{w} / \mathrm{v}$ ofloxacin solution) and the marketed formulation. The study revealed that ofloxacin was

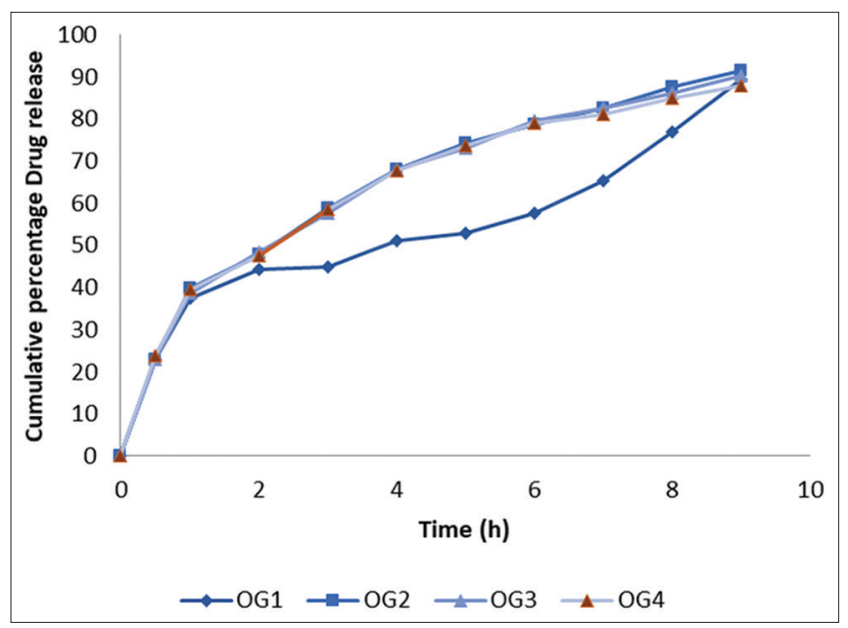

Fig. 1: In vitro drug release profile of ofloxacin from in situ gelling solutions

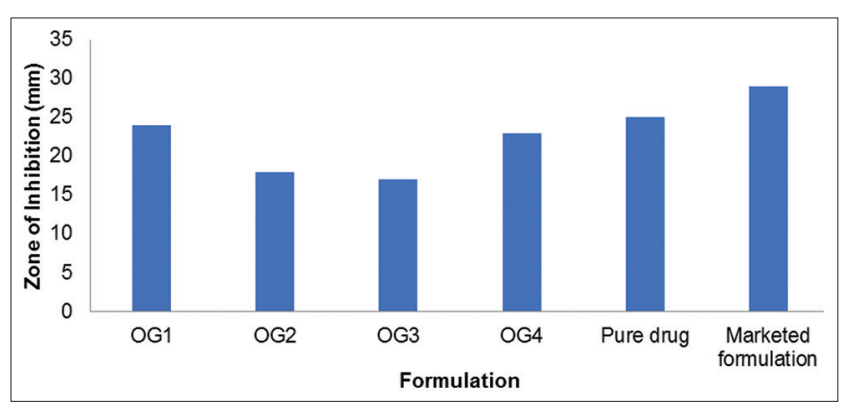

Fig. 2: Zone of inhibition of all the gel formulations, pure drug, and marketed formulation

Table 3: Results for $\mathrm{pH}$, gelling temperature, gelling capacity and drug content of drug incorporated polymer solutions

\begin{tabular}{llllll}
\hline Formulation code & Appearance & $\mathbf{p H}^{*}$ & Gelation temperature* & Gelling capacity & Drug content (\%)* \\
\hline OG1 & Light yellow & $6.9 \pm 0.4$ & $27 \pm 0.4$ & ++ & $96.74 \pm 0.23$ \\
OG2 & Light yellow & $7.15 \pm 0.05$ & $32 \pm 0.7$ & +++ & $95.17 \pm 0.45$ \\
OG3 & Light yellow & $7.2 \pm 0.15$ & $27.5 \pm 0.7$ & ++ & $95.76 \pm 0.34$ \\
OG4 & Light yellow & $6.9 \pm 0.3$ & $28.5 \pm 0.1$ & +++ & $94.67 \pm 0.32$ \\
\hline
\end{tabular}

*Mean and SD of $\mathrm{n}$ determinations; $\mathrm{n}=3$. SD: Standard deviation 
Table 4: Composition of developed in situ gel systems

\begin{tabular}{llll}
\hline Ingredients (g) & OG1 & OG2 & OG3 \\
\hline Ofloxacin & 0.075 & 0.075 & 0.075 \\
Poloxamer & 4 & 4 & 3.75 \\
HPMC 15 cps & - & 0.75 & 0.075 \\
PVA (Hot) & - & - & 4.25 \\
Sodium dihydrogen phosphate & 0.281 & -125 \\
Benzalkonium chloride (\% w/v) & 0.0025 & 0.281 & 0.281 \\
Phosphate buffer pH 7.4 & q.s & 0.0025 & 0.0025 \\
\hline
\end{tabular}

HPMC: Hydroxypropyl methyl cellulose, PVA: Polyvinyl alcohol, QS: Quantity Sufficient

Table 5: Results of kinetic analysis of drug release data

\begin{tabular}{llll}
\hline $\begin{array}{l}\text { Formulation } \\
\text { code }\end{array}$ & $\mathbf{R}^{2}$ & & \\
\cline { 2 - 4 } & $\begin{array}{l}\text { Zero order } \\
\text { model }\end{array}$ & $\begin{array}{l}\text { First order } \\
\text { model }\end{array}$ & $\begin{array}{l}\text { Higuchi matrix } \\
\text { model }\end{array}$ \\
\hline OG1 & 0.8732 & 0.9317 & 0.9397 \\
OG2 & 0.8764 & 0.9903 & 0.9591 \\
OG3 & 0.8715 & 0.9911 & 0.9898 \\
OG4 & 0.858 & 0.9869 & 0.9831 \\
\hline
\end{tabular}

effective even after formulation as an in situ gelling system as shown in Fig. 2.

\section{CONCLUSION}

Rapid elimination of the eye drops, administered often results in a short duration of the therapeutic effect which results in repeated administration. However the in situ gelling system may be a viable alternative to parenteral administration routes, thus improving patient compliance and may provide enhanced bioavailability compared with other delivery systems. The present work was taken up to use the solutions of the thermo-reversible polymer, Poloxamer 407 together, with mucoadhesive polymers such as PVA and HPMC to develop an in situ gelling system of ofloxacin which can be beneficial in overcoming the limitations of other administration routes.

The concentration of Poloxamer 407 chosen for the formulations had gelation below the temperature of the ocular cavity and was also, found to have a good gelling capacity. All the formulations showed higher amount of drug content, indicating that the method adopted for the formulation is suitable. The specific objectives were achieved for which further an extensive investigation is needed for the clinical usefulness of the developed in situ gels containing ofloxacin.

\section{ACKNOWLEDGMENT}

We are thankful to Sanofi Aventis, Goa for providing the drug sample and also to Nitte (Deemed to be University) for proving the facilities to carry out this work.

\section{AUTHORS' CONTRIBUTION}

The laboratory work has been carried out by the first author with inputs from the second author. The second author has edited the manuscript and is also the corresponding author.

\section{CONFLICTS OF INTEREST}

The authors declare that there are no conflicts of interest.

\section{REFERENCES}

1. Gupta S, Vyas SP. Carbopol/chitosan based $\mathrm{pH}$ triggered in situ gelling system for ocular delivery of timolol maleate. Sci Pharm 2010;78:959-76

2. Rhee YS, Park CW, DeLuca PP, Mansour HM. Sustained release injectable drug delivery. Pharm Technol 2010;2010 Suppl 6:1-7.

3. Talasaz AH, Ghahremankhani AA, Moghadam SH, Malekshahi MR, Atyabi F, Dinarvand R. In situ gel forming systems of poloxamer 407 and hydroxypropyl cellulose or hydroxypropyl methyl cellulose mixtures for controlled delivery of vancomycin. J Appl Polym Sci 2008;109:2369-74.

4. Bhoyar BS, Agnihotri VV, Bodhankar MM. New method for sustained ocular drug delivery: Based on polymeric combination with thermosensitive gelling agents. Int J Res Pharm Biomed Sci 2011;2:1151-60.

5. Rajasekaran A, Kumaran KS, Preetha JP, Karthika K. A comparative review on conventional and advanced ocular drug delivery formulations. Int J PharmTech Res 2010;2:668-74.

6. Qiu Y, Park K. Environment-sensitive hydrogels for drug delivery. Adv Drug Deliv Rev 2001;53:321-39.

7. Ron ES, Bromberg LE. Temperature-responsive gels and thermogelling polymer matrices for protein and peptide delivery. Adv Drug Deliv Rev 1998;31:197-221.

8. Bhalerao AV, Lonkar SL, Deshkar SS, Shirolkar SV, Deshpande AD, Nasal mucoadhesive in situ gel of ondansetron hydrochloride. Indian J Pharm Sci 2009;71:711-3.

9. Gupta H, Velpandian T, Jain S. Ion-and $\mathrm{pH}$-activated novel in-situ gel system for sustained ocular drug delivery. J Drug Target 2010;18:499-505.

10. Shastri DH, Prajapati ST, Parikh RK, Patel LD. Studies on poloxamer based muco-adhesive ophthalmic in situ hydrogel of moxifloxacin hydrochloride. Int J Pharm Res 2009;1:77-86.

11. Miyazaki S, Nakamura T, Takada M. Thermo-sensitive sol- gel transition of pluronic F- 127. Yakuzaigaku 1991;51:36-43.

12. Majithiya RJ, Ghosh PK, Umrethia ML, Murthy RS. Thermoreversiblemucoadhesive gel for nasal delivery of sumatriptan. AAPS PharmSciTech 2006;7:67.

13. Hiremath SS, Nadaf A, Dasankoppa FS, Jamakandi VG, Mulla JS, Sreenivas SA. Formulation and evaluation of a novel in situ gum based ophthalmic drug delivery system of linezolid. Sci Pharm 2008;76:515-32.

14. Kadam A, Jadhav R, Salunke P, Kadam S. Design and evaluation of modified chitosan based in situ gel for ocular drug delivery. Int J Pharm Pharm Sci 2017:9:87-1.

15. Mohan E, Kuri JK, Allenki V. Preparation and evaluation of $i n$-situ gel for ocular drug delivery. J Pharm Res 2009;2:1089-94.

16. Rajendran S, Ramesh S, Kumar S, Rao S. In vitro release kinetics of simvastatin from methyl cellulose gel. Int J Pharm Pharm Sci 2015;7:106-10. 